\title{
Rapid Mechanically Controlled Rewiring of Neuronal Circuits
}

\author{
Margaret H. Magdesian, ${ }^{1,2 \star}$ G. Monserratt Lopez-Ayon, ${ }^{1 *}{ }^{\top}$ Megumi Mori, ${ }^{1}$ Dominic Boudreau,,${ }^{3,4}$ \\ DAlexis Goulet-Hanssens, ${ }^{5}$ Ricardo Sanz, ${ }^{2}$ Yoichi Miyahara, ${ }^{1}$ Christopher J. Barrett, ${ }^{3,4}{ }^{\circledR}$ Alyson E. Fournier, ${ }^{2}$ \\ Yves De Koninck, ${ }^{3,4,6}$ and $\odot$ Peter Grütter ${ }^{1}$ \\ ${ }^{1}$ Department of Physics, McGill University, Montreal, Quebec H3A 2T8, Canada, ${ }^{2}$ Department of Neurology and Neurosurgery, Montreal Neurological \\ Institute, Montreal, Quebec H3A 2B4, Canada, ${ }^{3}$ Institut Universitaire en Santé Mentale de Québec, Quebec, Quebec G1J 2G3, Canada, ${ }^{4}$ Department of \\ Psychiatry and Neuroscience, Université Laval, Quebec, Quebec G1V 0A6, Canada, ${ }^{5}$ Department of Chemistry, McGill University, Montreal, Quebec H3A 2K6, \\ Canada, and ${ }^{6}$ Department of Pharmacology and Therapeutics, McGill University, Montreal, Quebec H3A 1Y6, Canada
}

CNS injury may lead to permanent functional deficits because it is still not possible to regenerate axons over long distances and accurately reconnect them with an appropriate target. Using rat neurons, microtools, and nanotools, we show that new, functional neurites can be created and precisely positioned to directly (re)wire neuronal networks. We show that an adhesive contact made onto an axon or dendrite can be pulled to initiate a new neurite that can be mechanically guided to form new synapses at up to $0.8 \mathrm{~mm}$ distance in $<1 \mathrm{~h}$. Our findings challenge current understanding of the limits of neuronal growth and have direct implications for the development of new therapies and surgical techniques to achieve functional regeneration.

Key words: atomic force microscopy; axonal growth; electrophysiology; neurodegenerative disease; neuronal networks; neuronal regeneration

\section{Significance Statement}

Brain and spinal cord injury may lead to permanent disability and death because it is still not possible to regenerate neurons over long distances and accurately reconnect them with an appropriate target. Using microtools and nanotools we have developed a new method to rapidly initiate, elongate, and precisely connect new functional neuronal circuits over long distances. The extension rates achieved are $\geq 60$ times faster than previously reported. Our findings have direct implications for the development of new therapies and surgical techniques to achieve functional regeneration after trauma and in neurodegenerative diseases. It also opens the door for the direct wiring of robust brain-machine interfaces as well as for investigations of fundamental aspects of neuronal signal processing and neuronal function.

\section{Introduction}

During development, neurons may extend axons over substantial distances to reach an appropriate target and build the intricate

Received April 29, 2015; revised Nov. 18, 2015; accepted Nov. 30, 2015.

Author contributions: M.H.M., G.M.L.-A., C.J.B., A.E.F., Y.D.K., and P.G. designed research; M.H.M., G.M.L.-A., M.M., D.B., A.G.-H., R.S., and Y.M. performed research; M.H.M. and P.G. contributed unpublished reagents/analytic tools; M.H.M., G.M.L.-A., Y.D.K., and P.G. analyzed data; M.H.M., G.M.L.-A., and P.G. wrote the paper.

This work was supported by the following (to P.G.): Natural Sciences and Engineering Research Council of Canada (NSERC) Discovery Grant, McGill University, James McGill research stipend, Le regroupement québécois sur les matériaux de pointe — Fond de recherche du Québec — Nature et technologies, Canadian Institutes of Health Research Training Grant, and NSERC Collaborative Research and Training Experience. We thank Dr. Edward Ruthazer and Dr. Delphine Gobert for critical discussions, contributions to experimental design, and elaboration of the manuscript; Dr. David Oliver for contributions on experimental design, data analysis, and elaboration of the manuscript; Dr. William Paul for technical support with electrophysiology setup; Jean-François Desjardins for data analysis discussions; Xue Ying Chua for help with cell cultures; Dr. Celia Greenwood for valuable discussions on data analysis and statistics; Dr. Paul De Koninck for helpful discussions on experimental design; Dr. Tim E. Kennedy and Stephen Glasgow for helpful discussions; and Robert Gagnon and John Smeros for technical support. functional networks that comprise the adult nervous system (Tessier-Lavigne and Goodman, 1996). The growth cone, a highly motile structure at the tip of the axon, interacts with guidance cues to accurately select a correct trajectory among multiple possible routes (Tessier-Lavigne and Goodman, 1996; Song and Poo, 2001; Dickson, 2002; Carmeliet and Tessier-Lavigne, 2005). Once the axon reaches its target, it arborizes and establishes synaptic connections, forming functional neural networks (Tessier-

The authors declare a potential conflict of interest. The devices and methods presented in this manuscript are protected by patent applications.

${ }^{*}$ M.H.M. and G.M.L.-A. contributed equally to this work.

Correspondence should be addressed to Professor Peter Grütter, Chair and James McGill Professor, Departmen of Physics, McGill University, 3600 University Street, Rm 108, Montreal, QC H3A 2T8, Canada. E-mail: peter.grutter@mcgill.ca.

DOI:10.1523/JNEUROSCI.1667-15.2016

Copyright $\odot 2016$ the authors $\quad 0270-6474 / 16 / 360979-09 \$ 15.00 / 0$ 
Lavigne and Goodman, 1996). Nervous system function depends on the precise specificity of neuronal connections.

Injuries to the adult CNS may lead to permanent disability due to the multiple mechanisms that limit axonal regrowth (Chew et al., 2012). Following injury, many CNS axons do not form a new growth cone and fail to mount an effective regenerative response (Bradke et al., 2012). Furthermore, damage and scar tissue surrounding CNS lesions significantly inhibit axonal growth (Ramon y Cajal, 1928; Aguayo et al., 1990; Tessier-Lavigne and Goodman, 1996; Horner and Gage, 2000; Song and Poo, 2001; Dickson, 2002; Carmeliet and Tessier-Lavigne, 2005; Yiu and He, 2006; Bradke et al., 2012; Chew et al., 2012). Current therapies to promote CNS regeneration after injury have focused on enhancing the intrinsic growth potential of the injured neuron and on masking the inhibitors of axon extension associated with myelin debris and the glial scar (Aguayo et al., 1990; Chew et al., 2012). Despite this, the capacity to regenerate long axons to distant targets and to form appropriate functional synapses remains severely limited. Santiago Ramon y Cajal's statement from 90 years ago remains essentially true today: "once the development was ended, the founts of growth and regeneration of the axons and dendrites dried up irrevocably. In the adult centers the nerve paths are something fixed, ended and immutable. Everything may die, nothing may be regenerated. It is for the science of the future to change, if possible, this harsh decree" (Ramon y Cajal, 1928).

In the present work, we use microbeads, atomic force microscopy (AFM), micromanipulation, and microfabrication techniques to rapidly initiate, elongate, and precisely connect new functional neuronal circuits over long distances. Earlier studies showed that cultured cerebellar neurons form presynaptic-like specializations when placed in contact with beads coated with positively charged proteins, including poly-L-lysine (Burry, 1980, 1982; Peng et al., 1987). Furthermore, previous work of our laboratory has shown that poly-D-lysine-coated beads (PDL beads) induce membrane adhesion followed by the clustering of synaptic vesicle complexes. Increased immunolabeling of synaptophysin, bassoon, rab3a interacting molecule, the N-type calcium channel CaV2.2, and N-cadherin, as well as rearrangement of actin and tubulin filaments, was observed at sites of contact between neurites and PDL beads, resulting in the formation of functional presynaptic boutons (Lucido et al., 2009). We also showed that when the PDL bead is mechanically pulled away after presynaptic differentiation, the synaptic protein cluster follows the bead, initiating a new neurite (Suarez et al., 2013). Transport of synaptophysin and bassoon were detected in the newly formed neurite (Suarez et al., 2013).

Here, we investigate the limits of extension and functionality of the new neurite. We show that new, functional neurites can be created and positioned to controllably (re)wire neuronal networks. The extension rates achieved are faster than $20 \mu \mathrm{m} / \mathrm{min}$ over millimeter-scale distances and functional connections are established. Our results show, unexpectedly, that the intrinsic capacity of these neurites for elongation is much faster than previously thought. Our proposed mechanical approach bypasses slow chemical strategies and enables controlled connection to a specific target. This technique opens new avenues for the in vitro study of novel therapies to restore neuronal connectivity after injury. It also enables the manipulation and rewiring of neuronal networks to investigate fundamental aspects of neuronal signal processing and neuronal function in vitro.

\section{Materials and Methods}

Microfluidic chambers. Microfluidic chambers, with microchannels to direct axonal extension or with isolated compartments to grow separated populations of neurons on the same dish, were designed and microfabricated by Advanced Nano-Design Applications, McGill University, Montreal, Quebec, Canada. These devices are made of polydimethylsiloxane (PDMS) using the Sylgard 184 Silicone elastomer kit (Dow Corning) as described previously (Magdesian et al., 2012). PDMS devices were assembled on $25 \mathrm{~mm}$ glass coverslips (Warner Instruments). The glass surface was coated with $100 \mu \mathrm{g} / \mathrm{ml}$ PDL (Sigma-Aldrich) and the devices were used to culture neurons.

Neuronal cultures. All procedures were approved by McGill University's Animal Care Committee and conformed to the guidelines of the Canadian Council of Animal Care. Hippocampal or cortical neurons from Sprague Dawley rat embryos of either sex (Charles River) were isolated as previously described (Lucido et al., 2009) and added to the microfluidic chambers. Cells were cultured in the microfluidic chambers for 14-21 d in vitro (DIV) and the PDMS devices were removed 1-4 d before AFM imaging or micromanipulation experiments as described previously (Magdesian et al., 2012). During experiments, cells were continuously perfused with physiological saline $[135 \mathrm{~mm} \mathrm{NaCl}$ (SigmaAldrich), $3.5 \mathrm{~mm} \mathrm{KCl}$ (Sigma-Aldrich), 2 mm CaCl2 (Sigma-Aldrich), 1.3 mм MgCl2 (BDH), 10 mm HEPES (ThermoFisher Scientific), and $20 \mathrm{~mm}$ D-glucose (Invitrogen); Goldman et al., 2013]. Osmolarity was 240-260 mOsm and $\mathrm{pH}$ was adjusted to 7.3-7.4 using $\mathrm{NaOH}$ (Sigma-Aldrich), with continuous bubbling of $\mathrm{O}_{2}$ to reduce $\mathrm{pH}$ oscillations during experiments.

AFM. Experiments were conducted using an MFP-3D-BIO AFM (Asylum Research) mounted on an Olympus IX-71 inverted optical microscope. The sample was placed in the closed fluid cell, open configuration, and was left undisturbed for $15 \mathrm{~min}$ to achieve thermal equilibrium at $37^{\circ} \mathrm{C}$. A $40 \times$-phase objective with 0.6 numerical aperture (Olympus) was used to focus on the sample, allowing optical access from the bottom and AFM access from the top of the sample. Triangular MLCT cantilevers (spring constant of $0.01 \mathrm{~N} / \mathrm{m}$; Bruker) were used and custom modified as described below. The region of interest was located and aligned with the cantilever tip using bright-field illumination.

Beading the AFM probe. A $50 \mu \mathrm{l}$ drop of either 4, 10, or $20 \mu \mathrm{m}$ beads (Polysciences) diluted in water (1:500) was deposited on a square coverslip and quickly dried at $37^{\circ} \mathrm{C}$. Epoxy adhesive (Loctite E-30Cl, Henkel) was added at one edge of another square coverslip and both coverslips were fixed at opposite sides of a microscope slide using vacuum grease 1 $\mathrm{cm}$ apart, with the dab of glue facing the center of the slide. The slide was positioned in the microscope. The tip of an AFM cantilever was brought in contact with the glue and retracted with a small droplet of glue on the tip. Next, the slide was shifted to the coverslip containing the beads. The AFM tip with glue was then brought in contact with the bead and lifted away. Bead attachment was optically confirmed and the glue was cured overnight at $37^{\circ} \mathrm{C}$.

AFM micromanipulation. Neurons were cultured for $14-21 \mathrm{~d}$ in microfluidic chambers. During experiments, cells were perfused with physiological saline. The sample was positioned in the AFM and a $10 \times$ objective used to find the region of interest. A $40 \times$-phase objective with 0.6 numerical aperture was used for optical measurements. The AFM beaded tip was brought in contact with a bundle of neurites for $30 \mathrm{~min}$, applying forces between 0.1 and $0.3 \mathrm{nN}$ (Magdesian et al., 2012). Next, the AFM tip was moved $5 \mu \mathrm{m}$ away from the sample at a speed of 0.5 $\mu \mathrm{m} / \mathrm{min}$, enabling the visualization of one or more neurites attached to the bead. The AFM tip was micromanipulated further at increasing speeds as long as intermittent periods of rest were allowed. Maximum speeds of $>100 \mu \mathrm{m} / \mathrm{min}$ were sustained, with average speeds of $20 \pm 10$ $\mu \mathrm{m} / \mathrm{min}$. We have yet to discover whether there is a fundamental upper limit to these speeds. After reaching the second target, the bead was brought in contact with the region of interest for $1 \mathrm{~h}$ and a force between 0.1 and $0.3 \mathrm{nN}$ was applied. After that the AFM was moved away from the sample. 

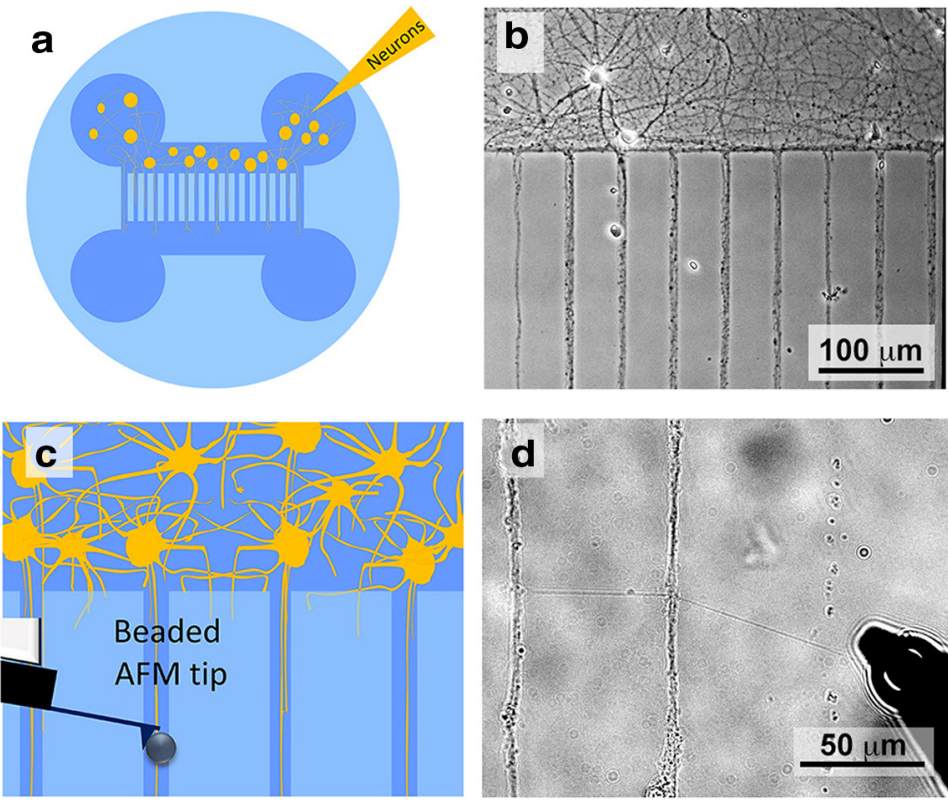

Figure 1. Initiation, elongation, and connection of new neurites in primary rat hippocampal neurons using PDL beads and AFM. $\boldsymbol{a}$, Design of the PDMS microfluidic microchamber (blue) assembled on a glass coverslip. $\boldsymbol{b}$, Neurons were cultured for 14-21 d in PDMS chambers enabling the growth of axons and dendrites inside the microchannels (Magdesian et al., 2012). c, PDMS was removed, exposing the parallel neurites aligned $60 \mu \mathrm{m}$ from each other. $\boldsymbol{d}$, A PDL bead attached to the AFM tip was brought in contact with a bundle of neurites. After $30 \mathrm{~min}$, the bead was pulled, thereby initiating and elongating a new neurite, which is extended and precisely connected to the next bundle of neurites.

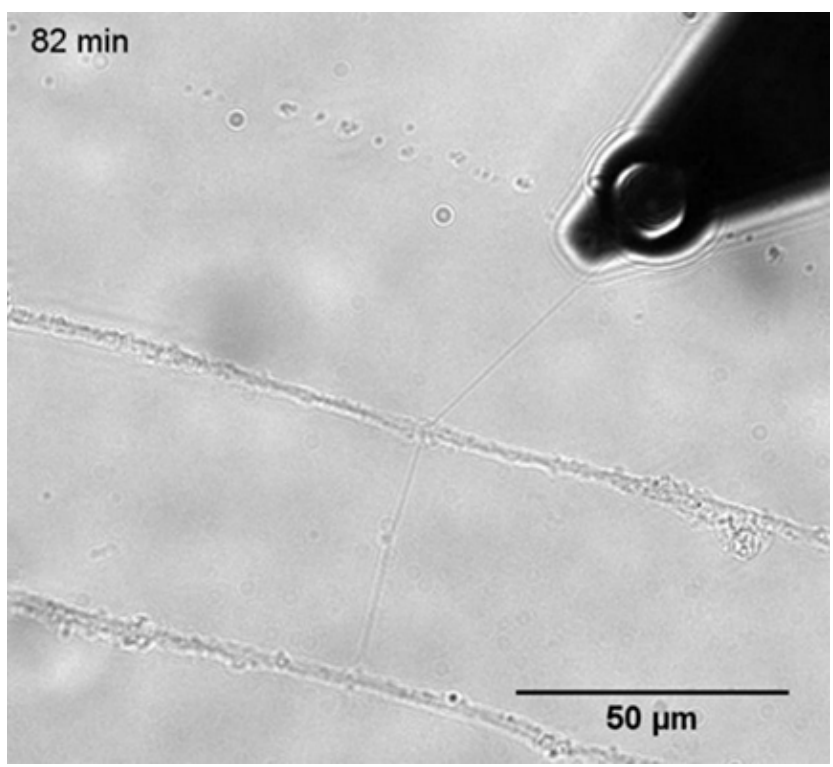

Movie 1. Initiation, elongation and connection of new neurites in primary rat hippocampal neurons using PDL beads and AFM. Neurons were cultured for 14-21 d in PDMS microfluidic chambers, enabling the growth of axons and dendrites inside the microchannels. PDMS was removed, exposing parallel neurites aligned $60 \mu \mathrm{m}$ from each other. A PDL bead attached to the AFM tip was brought in contact with a bundle of neurites. After $1 \mathrm{~h}$ in contact (time, $0 \mathrm{~min}$ ) the bead was pulled, thereby inducing and elongating a new neurite, which is extended and precisely connected to the next bundle of neurites (time, 0-18 $\mathrm{min}$ ). After $1 \mathrm{~h}$ in contact at the new site (time, $18-78 \mathrm{~min}$ ), and upon retraction of the cantilever, the new neurite remained attached to the bead and was elongated for $>840 \mu \mathrm{m}$ in $66 \mathrm{~min}$ (time, 78-144 min). Neurite elongation was limited by instrumentation restrictions since the experimental setup on the AFM does not allow displacements of $>1 \mathrm{~mm}$.
Pipette micromanipulation. Ten micrometer beads were coated with $100 \mu \mathrm{g} / \mathrm{ml} \mathrm{PDL}$ as described previously (Lucido et al., 2009), added to 14-21 DIV neurons grown in microfluidic chambers and incubated for $1 \mathrm{~h}$. Cell culture medium and nonadherent PDL-coated beads were removed and cells were positioned on an inverted optical microscope (Olympus IX-71) with perfusion of oxygenated physiological saline solution at room temperature at a rate of $0.5-1 \mathrm{ml} / \mathrm{min}$. Experiments were conducted using two pipette micromanipulators (MX7600R, SD Instruments; PCS-5000 Series, Burleigh). Pipettes with an outer tip diameter of $\sim 5 \mu \mathrm{m}$ were prepared from glass capillary tubes $(1.5 \mathrm{~mm}$ outer diameter; King Precision Glass). A $40 \times$-phase objective with 0.6 numerical aperture was brought into focus with the sample, allowing optical access from below and micromanipulation of the pipettes from above the sample. The PDL bead of interest was located using bright-field illumination. PDL beads attached to neurites along the edge of the neuronal population above the gap and close to neuronal cell bodies were selected. The tip of a pipette was optically guided with motorized micromanipulators close $(2-5 \mu \mathrm{m})$ to the target PDL bead. Negative pressure was applied with a $1 \mathrm{ml}$ syringe connected to the pipette to pull the PDL bead toward the pipette tip. Negative pressure in the pipette was maintained throughout the whole micromanipulation experiment to keep the PDL bead attached to the pipette. Next, the bead was moved $5 \mu \mathrm{m}$ away from the neurite at $0.5 \mu \mathrm{m} / \mathrm{min}$, initiating $\geq 1$ neurites attached to the bead. Subsequently, the PDL bead-neurite complex was pulled toward a neurite-dense area close to neuronal cell bodies in the neuronal population below the gap, extended at increasing speed as long as intermittent periods of rest were allowed, just as described for AFM micromanipulation. To ensure adhesion of the new neurite and the target, a PDL bead was positioned (with a second pipette) on top of the extended neurite and the target. After $1 \mathrm{~h}$, the PDL beads were released from the pipette tip and the new neurite(s) remained attached to the chosen target. Saline solution was replaced by Neurobasal medium (Invitrogen) and the sample was incubated overnight at $37^{\circ} \mathrm{C}$ with $5 \% \mathrm{CO}_{2}$ before electrophysiology tests were performed.

Immunocytochemistry. Immunocytochemistry was performed as previously described (Lucido et al., 2009) with mouse anti-tubulin (Developmental Studies Hybridoma Bank, University of Iowa), rabbit antineurofilament 200 (Sigma-Aldrich), mouse anti-tau-1 antibody clone PC1C6 (Millipore), and Alexa 488-conjugated phalloidin (Invitrogen). The secondary antibodies used were rhodamine red anti-mouse IgG (Invitrogen) and Alexa Fluor 647 anti-rabbit IgG (Invitrogen). Samples were imaged with a $40 \times$ objective on an inverted optical microscope (Olympus IX-71) or with a Fluoview FV1000 laser scanning confocal microscope (Olympus) with a $60 \times$ PlanApo oil-immersion objective on an inverted microscope.

Electrophysiology. Whole-cell paired patch-clamp recordings were conducted on hippocampal neurons perfused with oxygenated physiological saline at room temperature for $30 \mathrm{~min}$ prior and during patchclamp experiments. Pipettes were prepared from glass capillary tubes (1.5 $\mathrm{mm}$ outer diameter with filament; King Precision Glass). The pipettes to patch on the postsynaptic cell were filled with internal solution containing $95 \mathrm{~mm}$ Cs-methysulfonate (Sigma-Aldrich), $10 \mathrm{~mm}$ HEPES (ThermoFisher Scientific), $10 \mathrm{~mm} \mathrm{NaCl}$ (Sigma-Aldrich), $0.15 \mathrm{~mm}$ EGTA (Sigma-Aldrich), and $0.15 \mathrm{~mm} \mathrm{CaCl} 2$ (Sigma-Aldrich; Invitrogen; Goldman et al., 2013). The pipettes to patch on the presynaptic cell were filled with internal solution containing $95 \mathrm{~mm} \mathrm{~K}$-methysulfonate, $4 \mathrm{~mm} \mathrm{MgCl}_{2}$, and $10 \mathrm{~mm}$ HEPES (Trudel and Bourque, 2010). The $\mathrm{pH}$ of all internal solutions was adjusted to 7.2-7.3 using $\mathrm{CsOH}$ (Sigma-Aldrich) or $\mathrm{KOH}$ 

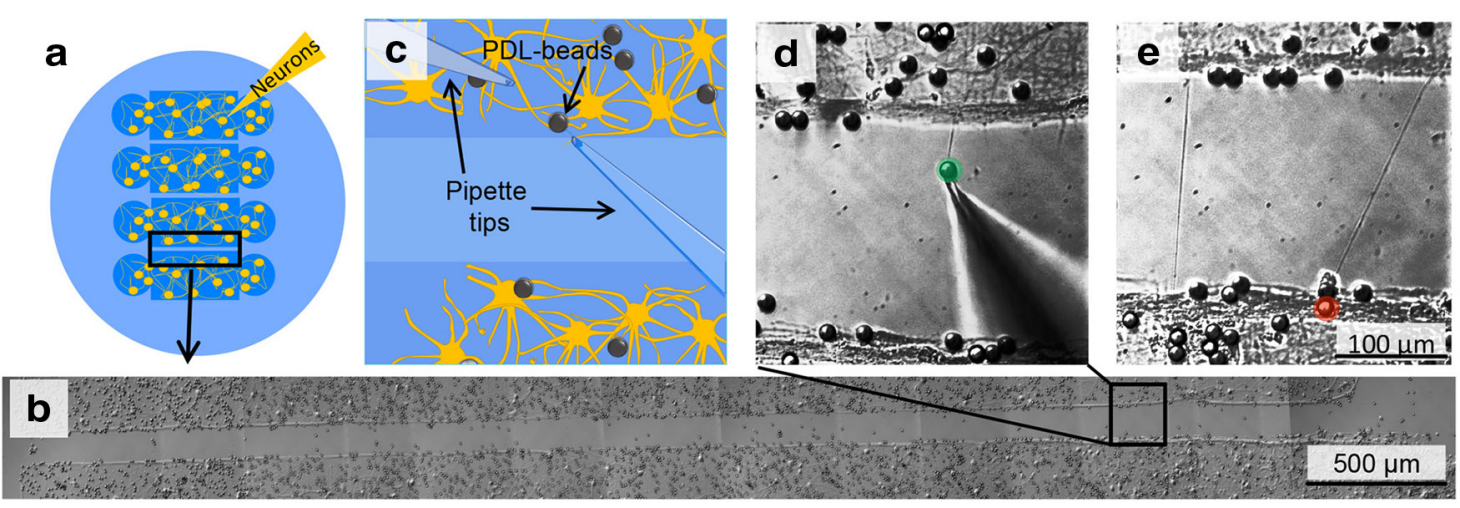

Figure 2. Initiation, elongation, and connection of new neurites using pipette micromanipulation. $\boldsymbol{a}$, Design of the microchamber (blue) assembled on a glass coverslip to grow dissociated cultures of rat primary hippocampal neurons in four isolated populations. $\boldsymbol{b}$, Before experiments were performed, the microchamber was disassembled, exposing the $200 \mu \mathrm{m}$ gap between neuronal populations (image composite shown), and neurons were incubated with PDL beads for $1 \mathrm{~h}$.c, Zoomed-in schematic representation of the experimental setup showing the gap between two isolated neuronal populations (population 1 above and population 2 below) as well as the position of the two pipette tips. $\boldsymbol{d}$, By applying negative pressure to a pipette, a PDL bead adhered to neuronal population 1 is pulled with the pipette tip, thereby initiating a new neurite. By maintaining the negative pressure in the pipette, the PDL bead-new neurite complex (green) can be pulled, enabling neurite elongation. $\boldsymbol{e}$, Pipette micromanipulation guides the extension $(>250 \mu \mathrm{m})$ of the new neurite over the gap and the formation of a connection with the neuronal population 2 . To ensure adhesion of the new neurite to neuronal population 2, a PDL bead (red) is positioned (with a second pipette) on top of the extended neurite and neuronal population 2 . Image shows two newly induced neurites guided with micromanipulation to connect two previously isolated neuronal populations in $<2 \mathrm{~h}$.

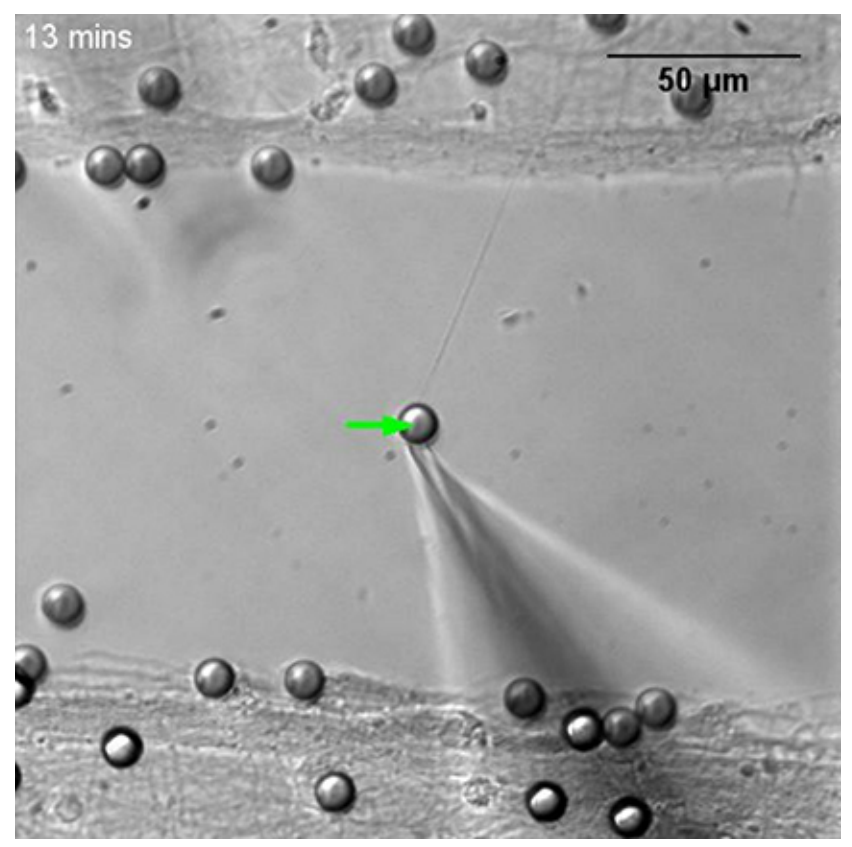

Movie 2. Initiation, elongation, and connection of new neurites in primary rat hippocampal neurons using PDL beads and pipette micromanipulation. We designed microchambers to grow dissociated neuronal cultures in isolated populations separated by a $200 \mu \mathrm{m}$ gap. Before experiments were performed, the microchamber was disassembled, exposing the gap between isolated neuronal populations 1 (above) and population 2 (below). By applying negative pressure to a pipette, a PDL-bead adhered to neuronal population 1 is pulled with the pipette tip, thereby initiating a new neurite. By maintaining the negative pressure in the pipette, the PDL bead-new neurite complex (green arrow) can be pulled, enabling neurite elongation. Pipette micromanipulation guides the extension ( $>250 \mu \mathrm{m}$ ) of the new neurite over the gap and the formation of a connection with the neuronal population 2. To ensure adhesion of the new neurite to neuronal population 2, a PDL bead (red arrow) is positioned (with a second pipette) on top of the extended neurite and neuronal population 2. Extension of the newly induced neurites to connect two previously isolated neuronal populations occurs in $<1 \mathrm{~h}$.

(Sigma-Aldrich) and the osmolarity was adjusted to $230-240 \mathrm{mOsml} / \mathrm{L}$. Pipette resistance in solution was 3-5 $\mathrm{M} \Omega$.

Cells were observed using bright-field illumination through $10 \times$ and $40 \times$ objectives. The electrodes were visually guided to the cell of interest using motorized micromanipulators. Images were captured using a cooled Cascade II camera mounted on a side port of the microscope and stored using Image Pro 6.2 software. The exposure time was set to $50 \mathrm{~ms}$, enabling live imaging at $\sim 20$ frames/s. Image stacks were acquired at a rate of $1 \mathrm{image} / \mathrm{min}$. Brightness and contrast were optimized to enable proper observation of the pipette-cell contact during approach and seal formation. Whole-cell recording was first established on one of the neurons in population 2 (postsynaptic cell), then the second pipette was sealed to the neuron in population 1 (presynaptic cell).

Once in whole-cell recording mode, the presynaptic cell was switched to current clamp and the baseline current was increased by $5-10 \mathrm{pA}$ to bring the cell closer to action potential threshold and to record stimulated action potentials. At the same time, the postsynaptic cell stayed in voltage clamp at a holding potential of $-70 \mathrm{mV}$ for recording of EPSCs. Paired patch-clamp recordings were performed using Axopatch-200A and Axopatch-200B amplifiers (Molecular Devices). Membrane current and voltage were digitized via an USB-6218 (National Instruments) coupled to a personal computer running WinWCP V4.7.3 software (University of Strathclyde Faculty of Science). WinWCP controlled the electrical stimuli provided. The signals were filtered at $5 \mathrm{kHz}$ through the amplifier and sampled at $10 \mathrm{kHz}$. Presynaptic and postsynaptic traces were continuously recorded. The paired recording data were exported as a text file for off-line analysis.

Data analysis. Matlab (Mathworks) was used to analyze the acquired image stacks as well as the paired patch-clamp recording traces. The total length of the neurites pulled was measured. The paired recordings were divided in intervals with a linear background. A background line was fitted and subtracted and a threshold voltage (for the presynaptic trace, $50 \mathrm{mV}$ ) or current (postsynaptic trace, $-40 \mathrm{pA}$ ) was selected. Presynaptic action potentials (PAPs) were detected as peaks higher than the threshold in the presynaptic trace, while EPSCs were detected as peaks lower than the threshold in the postsynaptic trace and within a $\pm 100 \mathrm{~ms}$ epoch from every PAP.

EPSCs were detected before or after PAP within $100 \mathrm{~ms}$ intervals and were classified into four categories: (1) EPSCs detected before and after PAP $(1,1)$; (2) EPSCs detected before PAP, but not after $(1,0)$; (3) EPSCs detected after PAP, but not before $(0,1) ;(4)$ no EPSCs detected within 100 ms interval of the PAP $(0,0)$.

All statistical analyses were performed with $\mathrm{R}$ project software and are based on logistic regressions for the rate of firing responses. First, a logistic regression containing a factor for experiment (not connected, naturally connected, or mechanically connected) as well as a factor for timing (comparing the $100 \mathrm{~ms}$ before the PAP with the $100 \mathrm{~ms}$ after the PAP) that was nested in the experiments was used to estimate not only whether the firing response rates were different between experiments, 

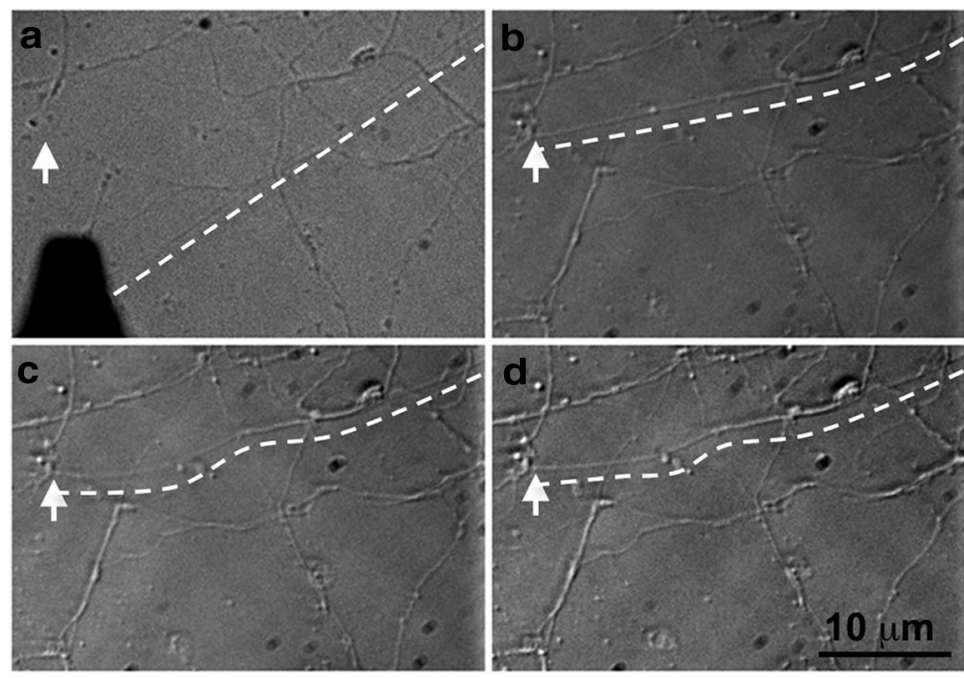

Figure 3. Micromanipulated connection of a new neurite to a distant cell is stable for $>24 \mathrm{~h}$. $\boldsymbol{a}, \boldsymbol{b}$, In a dissociated neuronal culture, a new neurite attached to a PDL bead at the AFM tip (a) was elongated and connected to another neurite (arrow) $>50 \mu \mathrm{m}$ apart (b). c, d, Images taken 12 (c) and $24 \mathrm{~h}(\boldsymbol{d})$ after connection show that the connection is stable and with time the initially straight and tense neurite $(\boldsymbol{b})$ becomes curved and relaxed ( $\boldsymbol{c}$ and $\boldsymbol{d}$ ). Arrows indicate the connection point and dashed lines highlight the new neurite.

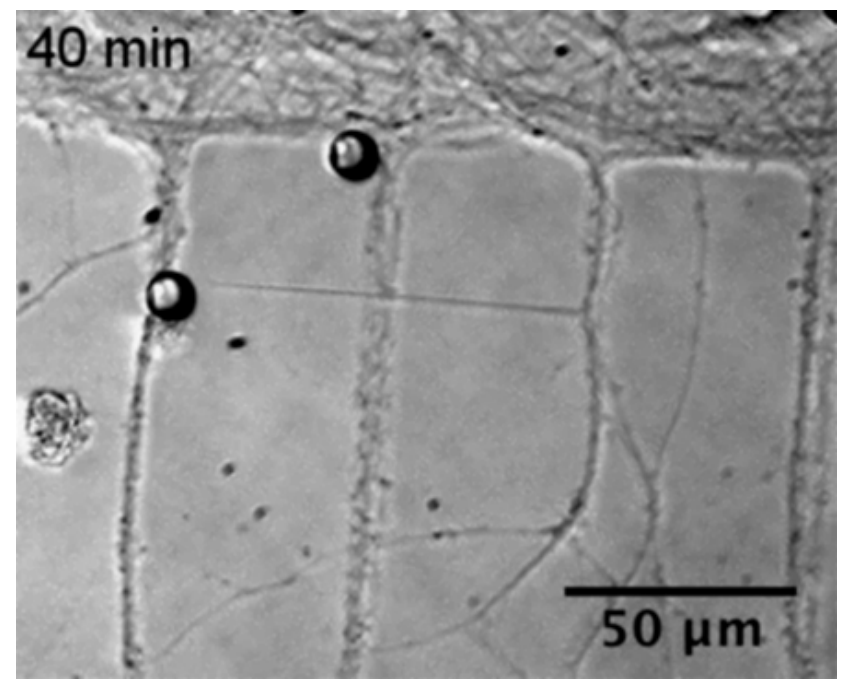

Movie 3. Initiation, elongation, and connection of new neurites using pipette micromanipulation. Neurons were cultured in PDMS microfluidic chambers, enabling the growth of axons and dendrites in parallel inside the microchannels for 14-21d. After PDMS removal, PDL beads were added to the culture and incubated for $1 \mathrm{~h}$. By applying negative pressure to a pipette, a PDL bead adhered to a bundle of axons and dendrites is pulled and attaches to the pipette tip, thereby initiating a new neurite. By maintaining the negative pressure in the pipette, the new neurite is elongated $>120 \mu \mathrm{m}$ and precisely guided to contact another bundle of axons and dendrites. After $1 \mathrm{~h}$ in contact, the bead is released from the pipette and the micromanipulated connection remains stable.

but also whether the firing rates were higher after the PAP rather than before, separately by experiment. Second, a logistic regression including terms for timing, experiment, and their interaction tested whether the timing effects showed evidence of differences across the experiments. The mean \pm SE were plotted as described previously (Xu et al., 2010). All differences were considered significant at $p<0.05$.

Spike-triggered activity is shown as a plot of 10 consecutive traces; the peak of every action potential was aligned at $0 \mathrm{~s}$ and the simultaneous postsynaptic trace was plotted. The onset of PAPs was located on average $5 \mathrm{~ms}$ before the AP peak and $5 \mathrm{mV}$ from baseline. The delay between the PAP and every postsynaptic peak was calculated within an interval of $\pm 100 \mathrm{~ms}$ to allow detection of events longer than monosynaptic activity $(\sim 5 \mathrm{~ms})$ and as much as $\sim 3$ synaptic delays apart $(\sim 20 \mathrm{~ms}$ each) since the probability of observing longer latencies is very low (Müller et al., 1997).

\section{Results}

We used rat hippocampal neurons grown in microfluidic devices, combined with AFM and optical microscopy, to manipulate neurite growth, synapse formation, and connectivity (Fig. 1). We attached a PDL bead to the tip of an AFM cantilever and precisely positioned the bead on top of bundles of neurites that grew inside the microchannels. AFM enables the precise control of the position and force applied by the bead on the neurites, avoiding neurite compression and consequent degeneration (Binnig et al., 1986; Magdesian et al., 2012). Once the PDL bead adhered to the first bundle of axons and dendrites, we used the AFM to pull the bead, thereby mechanically inducing the growth of a new neurite (Fig. 1c). We extended the new neurite for $\sim 60 \mu \mathrm{m}$ and put it in contact with the next bundle of axons and dendrites. After $30 \mathrm{~min}$, a stable connection was formed (Fig. 1d) and the new neurite was extended further for several hundred micrometers (Movie 1). During initial neurite extension, it was crucial to pull the neurite-PDL bead complex at a controlled rate of $0.5 \mu \mathrm{m} / \mathrm{min}$. Faster elongation rates during the first $5 \mu \mathrm{m}$ resulted in neurite rupture. Once the neurite was $\geq 5 \mu \mathrm{m}$ long, it could be elongated at an average speed of $20 \pm 10 \mu \mathrm{m} / \mathrm{min}$ up to a distance of $840 \mu \mathrm{m}$ (limited by instrumentation).

The number of neurites pulled with the bead depended on the thickness of the original neurite bundle that grew in the microchannel and on the bead size. Usually one neurite was pulled from bundles with a diameter of $<1.25 \pm 0.25 \mu \mathrm{m}$, while $\geq 3$ neurites could be pulled from bundles with a diameter of $>2.6 \pm$ $0.25 \mu \mathrm{m}$. We used three different sizes of beads: $4.5 \mu \mathrm{m}$ beads created one neurite in $60 \%$ of the experiments and two neurites in $20 \%(n=5)$, while $10 \mu \mathrm{m}$ beads pulled two filaments in $50 \%$ of the cases $(n=30)$ and $20 \mu \mathrm{m}$ beads pulled three neurites in $67 \%$ of the experiments and one neurite in only $11 \%$ of the experiments performed $(n=9)$.

Next, we conducted a series of experiments to determine whether the new neurite-PDL bead complex could be mechanically manipulated and guided to establish a functional connection with a selected target. We were able to initiate and extend a new neurite from a single neuron and precisely connect to a second neuron several hundreds of micrometers apart in $<2 \mathrm{~h}$. The newly formed connection was stable for $\geq 24 \mathrm{~h}$. To test the functionality and the type of connection, we put together an electrophysiology setup that enabled paired recordings. We faced several instrumental and biological challenges in performing paired recordings after the formation of the micromanipulated connection. Most neurons died or the newly formed connection was broken when we tried to perform whole-cell patch clamp on the same neuron that had just been elongated and connected.

To overcome these limitations, increase experimental yield, and test the functionality of the newly created neurites and connections, we decided to connect two individual neuronal popu- 

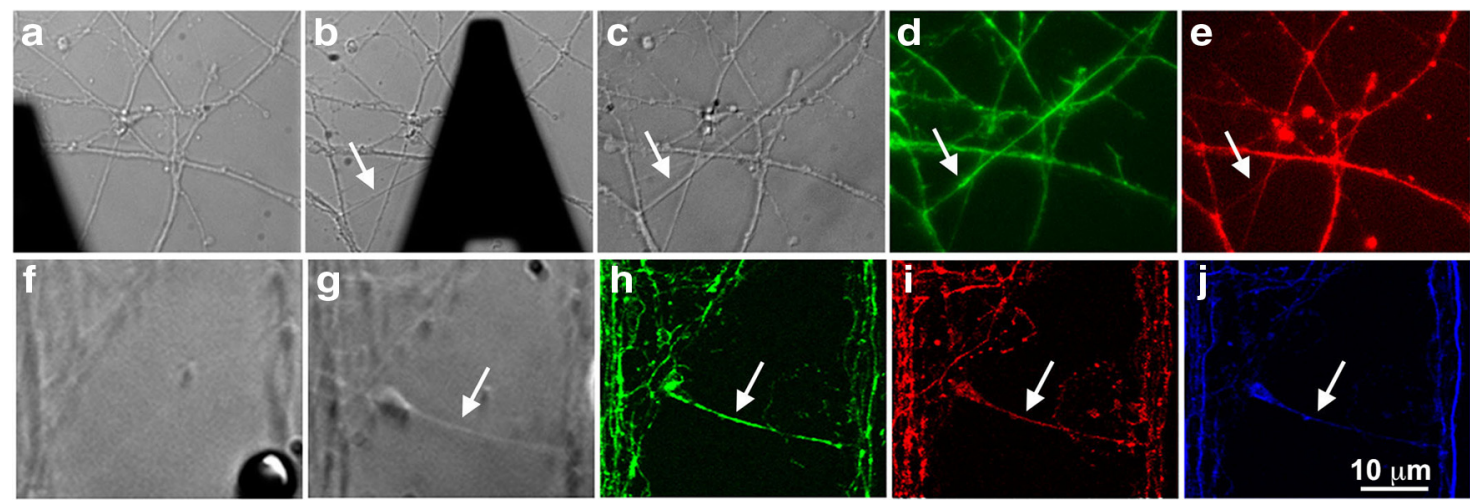

Figure 4. The newly formed neurite contains actin, tubulin, tau, and neurofilament. $\boldsymbol{a}, \boldsymbol{b}$, Using AFM micromanipulation, a neurite was contacted ( $\boldsymbol{a}$ ) and a new neurite was induced and extended (b). $\boldsymbol{c}-\boldsymbol{e}$, One hour after connection (c), cells were fixed and labeled with phalloidin (d) and anti-b-tubulin antibody $(\boldsymbol{e})$. $\boldsymbol{f}, \boldsymbol{g}$, Using pipette micromanipulation, a new neurite was induced $(\boldsymbol{f})$, extended, and connected $(\boldsymbol{g}) \cdot \boldsymbol{h}-\boldsymbol{j}$, After $18 \mathrm{~h}$ at $37^{\circ} \mathrm{C}$ with $5 \% \mathrm{CO}_{2}$, cells were fixed and labeled with phalloidin ( $\boldsymbol{h}$ ) and antibodies anti-tau- 1 (i) and anti-neurofilament $200(\boldsymbol{j})$. White arrows indicate the new neurite.

lations instead of connecting two individual neurons. This way we could first manipulate a connection between the two populations, then stimulate any neuron on population A and perform whole-cell patch-clamp recordings on any neuron on population $\mathrm{B}$ to investigate signal transmission through the newly formed connection. To grow isolated neuronal populations, physically separated by a $200 \mu \mathrm{m}$ gap, we developed a new microfluidic device (Fig. 2). To overcome AFM instrumentation limitations ( $>1$ h set-up time and short horizontal range), we used pipette micromanipulation techniques to connect the two populations (Fig. 2; Movie 2).

Pipette micromanipulation was adapted to achieve the necessary force and position precision to extend, guide, and connect multiple neurites in $<2 \mathrm{~h}$. First, PDL beads were added to a neuronal culture. After adhesion, single PDL beads were captured by suction at the tip of a micropipette. By pulling the PDL bead, a new neurite was formed and the neurite-PDL bead complex was guided in $<1$ h over the $200 \mu \mathrm{m}$ gap toward the second isolated neuronal population. Next, the neurite-PDL bead complex was positioned on top of a region rich in dendrites and, after adhesion, the neurite-PDL bead complex was released by releasing the suction on the pipette (Movie 2).

The releasing step was quite challenging because tension on the newly formed neurite tended to pull the neurite-PDL bead back to the initiation point. Previous work from our laboratory has shown that PDL beads added on top of a contact between an axon and a dendrite significantly increases the chances of synapse formation in the contact point (Lucido et al., 2009). Therefore, we used one micromanipulator to keep the new neurite-PDL bead in contact with the second neuronal population, and used a second micromanipulator to position another PDL bead on top of the newly formed neurite, pressing down to make sure that a second contact point between the new neurite and the second neuronal population was established. The aims of this technique were to avoid retraction, guarantee adhesion of the new neurite to the second neuronal population, and increase the chances of synapse formation. Once the connection between two previously isolated neuronal populations was stable, the sample was incubated overnight at $37^{\circ} \mathrm{C}$.

We achieved instrumentation reliability, thus ensuring that experiments could be performed routinely and that the mechanically induced connection of the new neurite was stable for $>24 \mathrm{~h}$ (Fig. 3). The technique of manipulating PDL beads is robust: new neurites were induced and extended in $95 \%$ of the experiments performed $(n=103)$. We reproduced these experiments with both rat hippocampal $(n=80)$ and cortical $(n=23)$ neurons grown inside microdevices with channels (Fig. 1; Movie 3) as well as in regular dissociated cultures (Fig. 3). In low-density dissociated neuronal cultures, PDL beads were brought in contact with the longest neurite of a neuron (presumably an axon) to initiate a new neurite. The new PDL bead-neurite complex was extended and connected to a region with multiple neurites. These experiments demonstrated that we could connect two previously unconnected neuronal populations (Fig. 2).

To assess the structural components present in the new neurites, we used fluorescent dyes and immunofluorescence techniques. The new neurites contain the same cytoskeletal components as naturally grown neurites, including actin, tubulin, tau, and neurofilament (Fig. 4; Franze and Guck, 2010). Neurite pulling was thus compatible with concurrent extension of cytoskeletal components, suggesting the formation of a new, structurally appropriate (and potentially functional) neuronal extension.

Next, we investigated whether the newly formed neurite used to connect two isolated neuronal populations was functional and able to transmit electrical signals. Using pipette micromanipulation and PDL beads, we extended a neurite from one neuronal population and guided it across the gap to connect to neurites in an isolated second neuronal population (Fig. $5 a-c$ ). After incubation for $24 \mathrm{~h}$, we performed whole-cell patch-clamp recordings (Fig. $5 d-f$ ).

We selected a neuron in population 1 , located within a 100 $\mu \mathrm{m}$ radius from the site where the induced neurite was initiated to record PAPs. This neuron was considered the presynaptic cell. Postsynaptic excitatory or inhibitory activity was recorded from a neuron in population 2, on the other side of the gap, located within a $100 \mu \mathrm{m}$ radius of the micromanipulated connection (Fig. $5 g-i$ ). By using this approach, there is a high probability that the selected presynaptic and postsynaptic neurons were not connected by a single synapse but rather polysynaptically connected. Indeed, the variable long latencies ( $\geq 25 \mathrm{~ms}$ ) between PAPs and EPSCs across the gap are typical of polysynaptic connections (Bray, 1984; Lamoureux et al., 2002, 2011; Rocco-Donovan et al., 2011).

To detect events longer than monosynaptic activity $(\sim 5 \mathrm{~ms})$ and as much as $\sim 3$ synaptic delays apart ( $\sim 20 \mathrm{~ms}$ each $)$, we recorded signals within an interval of $\pm 100 \mathrm{~ms}$ from the PAP. Recordings derived from the mechanically induced connections were analyzed and compared with those from naturally 


\section{Micromanipulated connection}

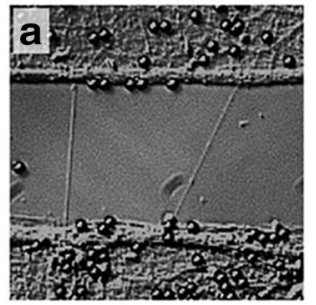

Natural connection

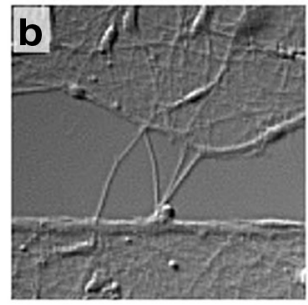

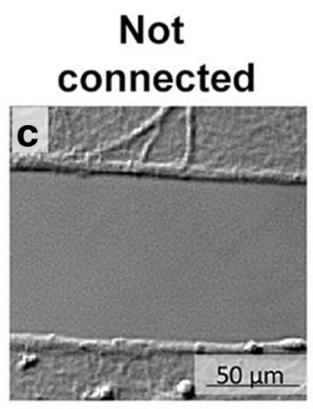
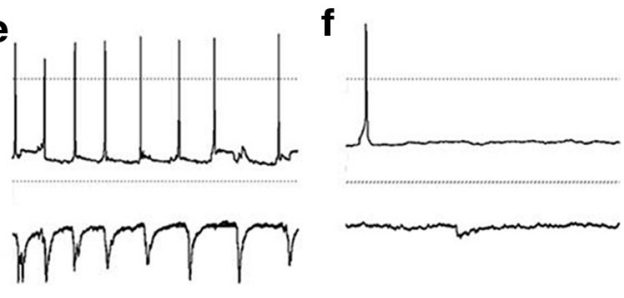

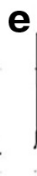

용

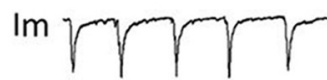

$5 \stackrel{00 \mathrm{~ms}}{ }$

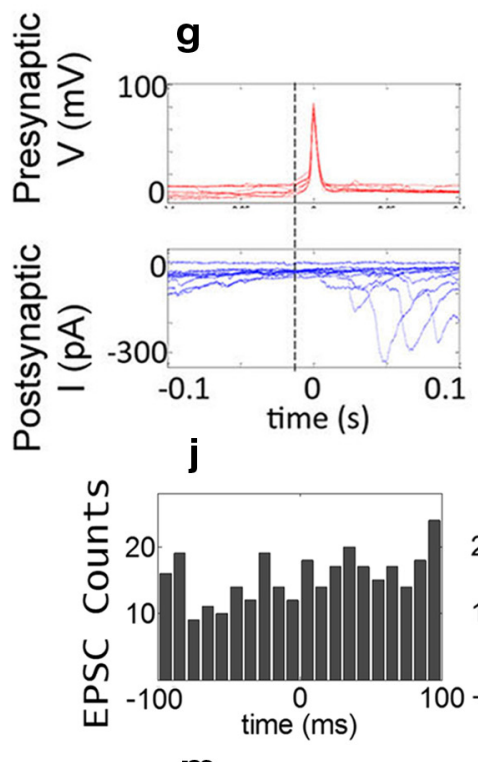

h

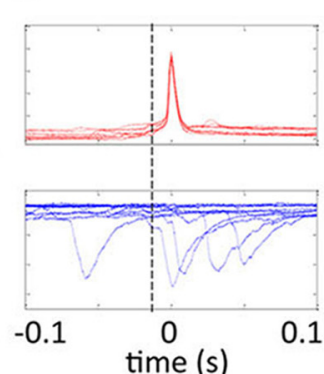

\section{$\mathbf{k}$}

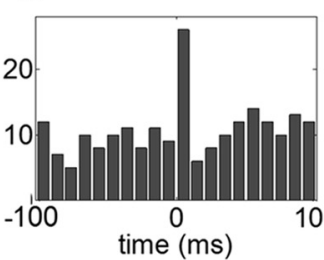

$100-100$

i

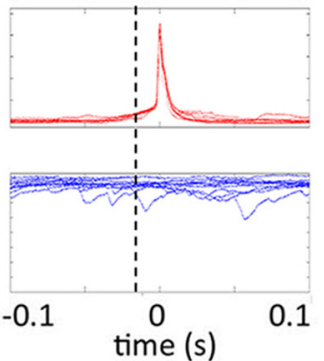

I

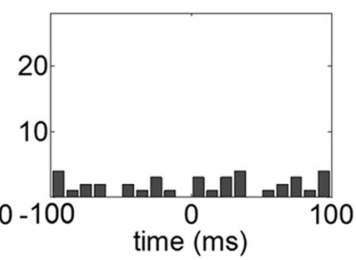

m

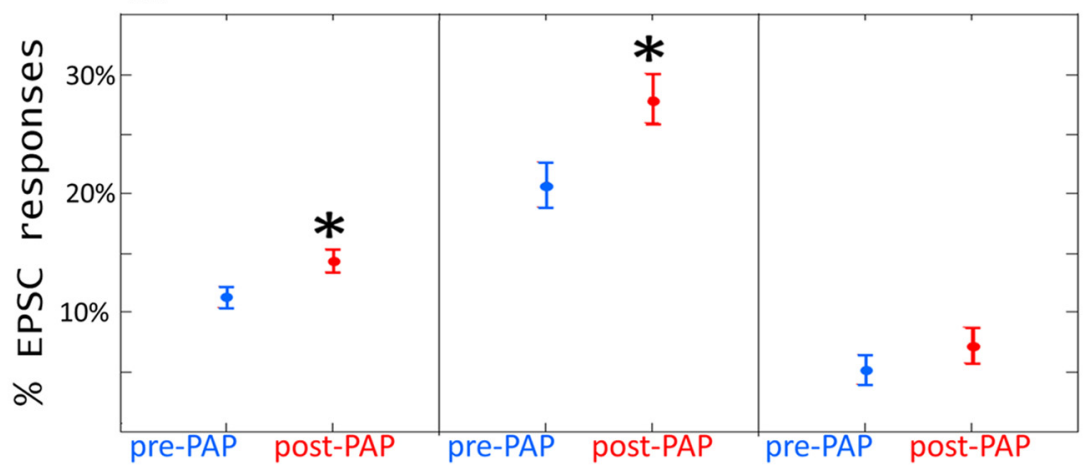

Figure 5. The newly induced, elongated, and connected neurite can transfer information between two isolated neuronal populations. Isolated neuronal populations were cultured, separated by a $100 \mu \mathrm{m}$ gap, in PDMS microdevices. $\boldsymbol{a}-\boldsymbol{c}$, Paired patch-clamp recordings were performed in whole-cell configuration from a neuron in population 1 and a neuron in population 2 (on the other side of the gap) when the two populations were connected through mechanical manipulation (a), allowed to naturally interconnect across the gap $(\boldsymbol{b})$, or remain nonconnected by maintaining the gap $(\boldsymbol{c}) \cdot \boldsymbol{d}-\boldsymbol{i}$, Representative traces of paired recordings ( $\boldsymbol{d}-\boldsymbol{f}$ ) and spike-triggered activity of 10 consecutive traces in an interval of $0-100 \mathrm{~ms}$ are shown for each condition $(\boldsymbol{g}-\boldsymbol{i})$. Dashed vertical lines $(\boldsymbol{g}-\boldsymbol{i})$ mark the onset of PAPs. The spike-triggered activity was analyzed and, for all the PAPs in each recording, the corresponding EPSCs were counted in the $\pm 100 \mathrm{~ms}$ interval. $j-I$, Counts of the EPSCs for each condition are shown in histograms. $\boldsymbol{m}$, The percentage of PAPs associated with postsynaptic responses in $\pm 100 \mathrm{~ms}$ intervals for each condition is

connected neuronal populations and nonconnected populations (Fig. 5). Table 1 summarizes PAP-EPSC counts for each condition.

Despite the low experimental yield for paired patch-clamp recordings $(<25 \%)$ and cell death after measurements (Debanne et al., 2008), we performed $>100$ connections of isolated neuronal populations and $>30$ paired patch-clamp recordings. Paired patch-clamp recordings were only performed in micromanipulated connections stable for $\geq 24 \mathrm{~h}$. The results presented in Figure 5 represent the analysis of the most stable recordings performed for $>1 \mathrm{~h}$ in the same pair of cells. It enabled the acquisition of $>1000$ PAP stimulations and sufficient data for statistical significant analysis. There is no difference in the age of the cultures used for the three experimental conditions. Each set of three experimental conditions was tested on the same day (DIV 15) in neuronal cultures from the same origin.

Analysis of PAPs and EPSCs using logistic regression (with timing nested in experiment) revealed that in the naturally connected group the number of EPSCs within a $100 \mathrm{~ms}$ epoch post-PAP was significantly higher than within a $100 \mathrm{~ms}$ epoch pre-PAP (Fig. $5 g-i ; p=0.012$ ). When the same analysis was performed in the mechanically connected group, we also observed an increase in the number of EPSCs $100 \mathrm{~ms}$ post-PAP (Fig. $5 g-i ; p=0.03$ ). No increase of EPSCs $100 \mathrm{~ms}$ post-PAP was observed in the nonconnected group (Fig. $5 g-i ; p=0.332$ ).

Counts of the EPSCs for each condition are shown in histograms (Fig. $5 j-l$ ). The rate of EPSCs within \pm 100 ms of the PAPs in the naturally versus the nonconnected samples was compared with logistic regression model. This analysis showed that the naturally connected sample had a significantly higher rate of responses than the nonconnected sample $(p=5 \mathrm{E}-8)$. The same analysis was performed to compare the mechanically connected versus nonconnected samples and the mechanically connected sample also had a significantly higher rate of responses than the nonconnected sample $(p=0.0005)$.

\section{$\leftarrow$}

shown. Data are the percentage \pm 28 SE for the mechanically induced connection $\left(n_{\text {pre-PAP }} / n_{\text {Total_PAP }}=136 / 1202\right.$, $\left.n_{\text {post-PAP }} / n_{\text {Total_PAP }}=173 / 1202\right)$, for neuronal populations naturally connected $\left(n_{\text {pre-PAP }} / n_{\text {Total_PAP }}=91 / 439, n_{\text {post-PAP }}\right.$ ) $\left.n_{\text {Total_PAP }}=123 / 439\right)$, and for unconnected populations $\left(n_{\text {pre-PAP }} / n_{\text {Total_PAP }}=16 / 304, n_{\text {post-PAP }} / n_{\text {Total_PAP }}=22 / 304\right)$. ${ }^{*} p<0.05$ as assessed by logistic regression (McCullagh, 1989). 
Table 1. Counts of PAPs and postsynaptic current peaks

\begin{tabular}{|c|c|c|c|}
\hline Conditions & Mechanically induced connection & Natural connection & Not connected \\
\hline 0,0 & $878(73 \%)$ & $225(51 \%)$ & $266(87 \%)$ \\
\hline 1,0 & $136(11 \%)$ & $91(20 \%)$ & $16(5 \%)$ \\
\hline 0,1 & $173(14 \%)$ & $123(28 \%)$ & $22(7 \%)$ \\
\hline 1,1 & $15(1 \%)$ & 0 & 0 \\
\hline Total number of PAPs & 1202 & 439 & 304 \\
\hline
\end{tabular}

PAPs with peak heights $>50 \mathrm{mV}$ from baseline were detected and counted in the presynaptic recording. Within a \pm 100 ms epoch from every PAP, EPSCs with absolute peak heights $>40 \mathrm{pA}$ from baseline were detected and counted. The data are organized in four categories: 0, 0: no EPSC detected $100 \mathrm{~ms}$ pre-PAP and no EPSC detected 100 ms post-PAP; 1,0 : EPSC(s) detected 100 ms pre-PAP, but no EPSC detected 100 ms post-PAP; $0,1:$ no EPSC detected $100 \mathrm{~ms}$ pre-PAP and EPSC(s) detected $100 \mathrm{~ms}$ post-PAP; 1 , 1: EPSC(s) detected $100 \mathrm{~ms}$ pre-PAP and EPSC detected $100 \mathrm{~ms}$ post-PAP. The percentage of the total number of PAPs is included for each of the categories.

When the mechanically connected sample was compared with the naturally connected, the rate of the responses was significantly higher for the naturally connected sample ( $p=4.3 \mathrm{E}-5)$. In addition, we performed a test of interaction between the experimental groups and the timing factors and saw no evidence of interaction $(p=0.8)$, indicating that each experimental group has a different response profile.

The electrical responses after PAPs recorded from neurons connected naturally and by micromanipulation are significantly higher and temporally correlate with the presynaptic activity (Fig. $5 g-m)$. Together, these results unequivocally indicate that functional connections were established by the direct micromanipulation of new synaptic connections.

Interestingly, the electrical responses to PAPs in the natural and micromanipulated connection are statistically different. Analysis of the EPSC counts show that the natural connection has a short-latency peak (the bin from 0 to $+0.01 \mathrm{~s}$ ) in firing probability (Fig. $5 h$ ), while there is little or no short-latency peak in the histogram of EPSCs for the mechanical connection (Fig. $5 g$ ). These findings are consistent with the data shown in Figure $5 g$, which shows medium to long latency, but no short-latency EPSCs for mechanical connections, and Figure $5 h$, which shows only short-latency and medium-latency EPSCs.

Differences between the electrical responses to PAPs for natural and mechanical connections could be due to different reasons: differences in neurite maturation and differences in number of functional synapses. In the micromanipulated connection, the newly induced neurite has been connected for only $24 \mathrm{~h}$, while in the natural connection the two populations have been connected and interacting for $\geq 10 \mathrm{~d}$. Therefore, new mechanically induced neurites may not be as mature or functional as the natural neurites, resulting in differences in conduction velocity and in synaptic transmission. In addition, differences in the number of synapses involved and path followed by the electrical stimuli in each neuronal population may also contribute to the differences between micromanipulated and natural connections. Our technique opens a new field for the study and comparison of natural and micromanipulated neuronal connections.

Paired whole-cell patch-clamp recording have very low experimental yield and cause cell death after measurements. To overcome these limitations, future studies will need new platforms to more precisely stimulate, record, and compare neuronal activity in natural and micromanipulated connections.

\section{Discussion}

This work shows for the first time that it is possible to create new neurites, elongate them for hundreds of micrometers in $<1 \mathrm{~h}$, and functionally connect the new neurites to any desired target to (re)wire neuronal networks. The great advantage of the current technique is that it is not necessary to wait for neurites to natu- rally grow, eventually interact with chemical cues, possibly turn toward a target, and maybe form functional connections. The present technique overcomes limitations found with previous approaches and offers high precision and control to induce the formation of a new neurite from a presynaptic bouton and mechanically extend new neurites at unprecedented speed to functionally connect to a chosen target.

This method has the potential to rapidly bridge gaps formed by neural scar tissue. Therapies for functional recovery after axonal injury have focused on biochemical cues to reproduce the sequence of events during axonal growth, namely growth cone assembly followed by axonal extension and synapse formation (Chew et al., 2012). Despite the development of promising new drugs, achieving long-distance axonal regeneration to establish functional connectivity that mimics the intact nervous system has remained a major challenge (Chew et al., 2012).

Previous studies have shown elongation of axons at 20-40 $\mu \mathrm{m} / \mathrm{h}$ by applying mechanical tension to an existing growth cone (Bray, 1984; Lamoureux et al., 1998, 2002, 2011; Pfister et al., $2004,2006)$. Here we show that new neurites can be created without a pre-existing growth cone and extended $\geq 60$ times faster than previously reported. We can extend new neurites at $0.5 \mu \mathrm{m} /$ min over the first $5 \mu \mathrm{m}$ followed by fast extension at an average speed of $20 \pm 10 \mu \mathrm{m} / \mathrm{min}$ up to distances of $840 \mu \mathrm{m}$ (limited only by instrumentation).

Axons undergoing slow stretch are structurally indistinguishable from those naturally grown (Pfister et al., 2004), suggesting that axonal growth is not limited by cellular components, but rather by limitations imposed by growth-cone performance, which exerts relatively little tension to exploit the full intrinsic capacity of axons to elongate (Lamoureux et al., 1998). In addition, stretched PNS neurons retain the ability to transmit active electrical signals (Pfister et al., 2004).

Our findings reveal that the newly created CNS neurites contain the main structural components of naturally grown neurites and are able to transmit electrical signals. To the best of our knowledge these measurements are the first micromanipulated neuronal connections. No data on electrical signal propagation through neuronal micromanipulated connections was found in the literature to compare with the results obtained here. Overall, the postsynaptic activity of the micromanipulated and natural connections is significantly higher compared with the nonconnected neuronal populations. Micromanipulated and natural connections show significantly more responses after than before the onset of the PAPs. In contrast, no significant increase was observed in nonconnected neuronal populations. The present work shows that it is possible to create and control new functional neuronal connections. Clearly more studies need to be done to understand whether and how micromanipulated connections differ from natural ones. We are currently investigating new, noninvasive electronic platforms as alternatives to whole-cell patch-clamp recordings to perform long-term high-throughput analysis of signal propagation in micromanipulated neuronal connections.

The technique presented here offers a new platform to obtain faster, more detailed and much more controlled data on how neurons communicate, how networks modulate signals, and how neurite growth and regeneration occurs. This method enables the systematic engineering of interconnection patterns in neuronal networks, allowing the creation of reproducible topologies in vitro. Also, this method, because it enables control of the length and diameter of the induced neurite and the easy generation of a predefined, controlled neuronal connectivity or topology, could 
be used to study signal propagation models (Maia and Kutz, 2014) and to transfer functions of neuronal networks. Our approach may also facilitate the development of robust brainmachine interfaces by connecting functional neurites onto inorganic interfaces or artificially building the 7000 synapses between the 302 neurons of the $C$. elegans nervous system to reproduce its connectome (White et al., 1986; Varshney et al., 2011; Jarrell et al., 2012), enabling a systematic investigation of the functional relevance of this known topology.

\section{References}

Aguayo AJ, Bray GM, Rasminsky M, Zwimpfer T, Carter D, Vidal-Sanz M (1990) Synaptic connections made by axons regenerating in the central nervous system of adult mammals. J Exp Biol 153:199-224. Medline

Binnig G, Quate CF, Gerber C (1986) Atomic force microscope. Phys Rev Lett 56:930-933. CrossRef Medline

Bradke F, Fawcett JW, Spira ME (2012) Assembly of a new growth cone after axotomy: the precursor to axon regeneration. Nat Rev Neurosci 13: 183-193. CrossRef Medline

Bray D (1984) Axonal growth in response to experimentally applied mechanical tension. Dev Biol 102:379-389. CrossRef Medline

Burry RW (1980) Formation of apparent presynaptic elements in response to poly-basic compounds. Brain Res 184:85-98. CrossRef Medline

Burry RW (1982) Development of apparent presynaptic elements formed in response to polylysine coated surfaces. Brain Res 247:1-16. CrossRef Medline

Carmeliet P, Tessier-Lavigne M (2005) Common mechanisms of nerve and blood vessel wiring. Nature 436:193-200. CrossRef Medline

Chew DJ, Fawcett JW, Andrews MR (2012) The challenges of long-distance axon regeneration in the injured CNS. Prog Brain Res 201:253-294. CrossRef Medline

Debanne D, Boudkkazi S, Campanac E, Cudmore RH, Giraud P, FronzaroliMolinieres L, Carlier E, Caillard O (2008) Paired-recordings from synaptically coupled cortical and hippocampal neurons in acute and cultured brain slices. Nat Protoc 3:1559-1568. CrossRef Medline

Dickson BJ (2002) Molecular mechanisms of axon guidance. Science 298: 1959-1964. CrossRef Medline

Franze K, Guck J (2010) The biophysics of neuronal growth. Rep Prog Phys 73:094601. CrossRef

Goldman JS, Ashour MA, Magdesian MH, Tritsch NX, Harris SN, Christofi N, Chemali R, Stern YE, Thompson-Steckel G, Gris P, Glasgow SD, Grutter P, Bouchard JF, Ruthazer ES, Stellwagen D, Kennedy TE (2013) Netrin-1 promotes excitatory synaptogenesis between cortical neurons by initiating synapse assembly. J Neurosci 33:17278-17289. CrossRef Medline

Horner PJ, Gage FH (2000) Regenerating the damaged central nervous system. Nature 407:963-970. CrossRef Medline

Jarrell TA, Wang Y, Bloniarz AE, Brittin CA, Xu M, Thomson JN, Albertson DG, Hall DH, Emmons SW (2012) The connectome of a decisionmaking neural network. Science 337:437-444. CrossRef Medline

Lamoureux P, Buxbaum RE, Heidemann SR (1998) Axonal outgrowth of cultured neurons is not limited by growth cone competition. J Cell Science 111:3245-3252. Medline

Lamoureux P, Ruthel G, Buxbaum RE, Heidemann SR (2002) Mechanical tension can specify axonal fate in hippocampal neurons. J Cell Biol 159: 499-508. CrossRef Medline

Lamoureux P, Heidemann S, Miller KE (2011) Mechanical manipulation of neurons to control axonal development. J Vis Exp pii:2509. CrossRef Medline

Lucido AL, Suarez Sanchez F, Thostrup P, Kwiatkowski AV, Leal-Ortiz S, Gopalakrishnan G, Liazoghli D, Belkaid W, Lennox RB, Grutter P, Garner CC, Colman DR (2009) Rapid assembly of functional presynaptic boutons triggered by adhesive contacts. J Neurosci 29:12449-12466. CrossRef Medline

Magdesian MH, Sanchez FS, Lopez M, Thostrup P, Durisic N, Belkaid W, Liazoghli D, Grütter P, Colman DR (2012) Atomic force microscopy reveals important differences in axonal resistance to injury. Biophys J 103:405-414. CrossRef Medline

Maia PD, Kutz JN (2014) Identifying critical regions for spike propagation in axon segments. J Comput Neurosci 36:141-155. CrossRef Medline

McCullagh P (1989) Generalized linear models. London: Chapman and Hall.

Müller TH, Swandulla D, Zeilhofer HU (1997) Synaptic connectivity in cultured hypothalamic neuronal networks. J Neurophysiol 77:3218-3225. Medline

Peng HB, Markey DR, Muhlach WL, Pollack ED (1987) Development of presynaptic specializations induced by basic polypeptide-coated latex beads in spinal cord cultures. Synapse 1:10-19. CrossRef Medline

Pfister BJ, Iwata A, Meaney DF, Smith DH (2004) Extreme stretch growth of integrated axons. J Neurosci 24:7978-7983. CrossRef Medline

Pfister BJ, Bonislawski DP, Smith DH, Cohen AS (2006) Stretch-grown axons retain the ability to transmit active electrical signals. FEBS Lett 580: 3525-3531. CrossRef Medline

Ramon y Cajal S (1928) Degeneration and regeneration of the nervous system. J Neurol Psychopathol 9:750.

Rocco-Donovan M, Ramos RL, Giraldo S, Brumberg JC (2011) Characteristics of synaptic connections between rodent primary somatosensory and motor cortices. Somatosens Mot Res 28:63-72. CrossRef Medline

Song H, Poo M (2001) The cell biology of neuronal navigation. Nat Cell Biology 3:E81-E88. CrossRef Medline

Suarez F, Thostrup P, Colman D, Grutter P (2013) Dynamics of presynaptic protein recruitment induced by local presentation of artificial adhesive contacts. Dev Neurobiol 73:98-106. CrossRef Medline

Tessier-Lavigne M, Goodman CS (1996) The molecular biology of axon guidance. Science 274:1123-1133. CrossRef Medline

Trudel E, Bourque CW (2010) Central clock excites vasopressin neurons by waking osmosensory afferents during late sleep. Nat Neurosci 13: 467-474. CrossRef Medline

Varshney LR, Chen BL, Paniagua E, Hall DH, Chklovskii DB (2011) Structural properties of the Caenorhabditis elegans neuronal network. PLoS Comput Biol 7:e1001066. CrossRef Medline

White JG, Southgate E, Thomson JN, Brenner S (1986) The structure of the nervous system of the nematode Caenorhabditis elegans. Philos Trans R Soc Lond B Biol Sci 314:1-340. CrossRef Medline

Xu B, Feng X, Burdine RD (2010) Categorical data analysis in experimental biology. Dev Biol 348:3-11. CrossRef Medline

Yiu G, He Z (2006) Glial inhibition of CNS axon regeneration. Nat Rev Neurosci 7:617-627. CrossRef Medline 